\title{
Analyzing the Rhetorical, Typographical and Paralinguistic Features of Electronic Mails in the Workplace
}

\author{
Mohammad Awad AlAfnan \\ Head of English Language Department, BMIC \\ Kuala Lumpur, Malaysia \\ Email: mohammad_alafnan@bmic.edu.my
}

Received: 27-11- 2014

Published: 01-07- 2015
Accepted: 01-02- 2015

doi:10.7575/aiac.ijalel.v.4n.4p.77
Advance Access Published: February 2015

URL: http://dx.doi.org/10.7575/aiac.ijalel.v.4n.4p.77

\begin{abstract}
This study investigated the rhetorical, typographical and paralinguistic features used in workplace emails. It revealed that the email exchanges included both spoken and written language features. The use of these features depended on two main factors that are the degree of involvement between the communicators, on the one hand, and the frequency of exchanging emails regarding a single issue on the other. This study also revealed that the communicative purpose of the email has prompted the type of features used in the emails. The emails that included tasks which were previously carried out using oral methods of communication in the workplace (i.e., face-to-face conversations, telephone calls) included several oral communication practices and typographical errors, whereas the emails that included tasks which were traditionally carried out using written method of communication in the workplace (i.e., letters, memorandums, faxes) mainly included written language features and were written appropriately.
\end{abstract}

Keywords: Email communication; Workplace communication; Rhetorical structures; Typographical features; Paralinguistic strategies

\section{Introduction}

The use of email for business communication has increased in the last decade not only in the Western economies but in the Eastern economies as well (AlAfnan, 2015a, 2015b). The use of email for organizational communication has become a daily practice that is inevitable, especially when organizations establish partnerships with international bodies or institutions (AlAfnan, 2014a, 2014b). Researchers have emphasized that the use of email and computer-mediated communication was driven by several organizational factors such as speed and low cost (Baruch, 2005; Case, 1996). However, it did not take long to realize that the use of email for business communication has changed the organizational and linguistic practices in the world of business. This use was generally theorized as hybrid that includes spoken and written forms (AlAfnan, 2015a, 2015b; Hale and Scanlon, 1999; Yates and Orlikowski, 1993). Baron (1998), for example, viewed the social dynamics of emails as predominantly writing, the lexicon and style as predominantly speech, whereas the format and syntax were seen as a mixture of writing and speech. This view, in fact, was shared by a number of researchers who mainly looked at the language used in emails that were exchanged in the business or corporate sector (Cassel and Tversky, 2005; Gains, 1999; Gimenez, 2000), however, examining the language used in email that were exchanged in the educational sector did not receive a lot of attention (AlAfnan, 2014a, 2014b). Having said that, this study investigates the discourse practices used in emails that were exchanged in one private educational institute in Malaysia.

According to Fairclough (1992), discourse practices refer to the practices used in producing, distributing and consuming the texts in a given context. By emphasizing the importance of the context, Fairclough has tinted that the use of discourse practices vary from a context to another. As such, identifying who wrote the text, why the text was written or for what purpose and to whom it was sent are all elements that need to be examined in order to comprehend the production and the consumption of the given texts. The importance of analyzing discourse practices emerges from the fact that they complement the textual analysis, as they emphasize the importance of textual features in the production and consumption of the texts (Palli et al., 2009). Discourse practice, according to Fairclough (1995, p. 60), "straddles the division between society and culture on the one hand, and discourse, language and text on the other". Having said that, this paper analyses the discourse practices in the emails focusing on the rhetorical structures, paralinguistic strategies, and the typographical features used in constructing the texts and how these strategies are interpreted in their context. The rhetorical structures include the usage of hedges, ellipsis, repetition, and substitution, whereas the paralinguistic strategies include the usage of emoticons, capitalizations, and different color, print and font choices. Typographical features, however, include decapitalization and spelling mistakes.

\section{Methods}

In order to collect the emails, the researcher approached seven full-time employees who work in CIF (a pseudonym), an educational Institute in Kuala Lumpur. The organizational position of the main informants varied from office assistants to the assistant academic director. They are three males and four females. Their educational background also varies from 
Diploma to Master degree. These seven main informants, in fact, were chosen after a deep observation of the rules played in the Institute by the full-time staff. They are key players in their organizational position, belong to the three main Malaysian ethnic backgrounds (Malay, Chinese, and Indians) and use email for workplace communication. As such, it is believed that the email messages that are provided by these informants would reflect and represent the use of emails in the Institute.

I explained to the informants the nature of the study and the required data. They agreed to provide their email exchanges and signing the consent form. It was agreed that the main informants would provide six weeks of day-in-day-out email communications. The emails were sent to the researcher either directly using the blind carbon copy 'BCC' option, or before signing off the working day from 4:45-5:00 pm. At the end of the collection period, the researcher received 522 email messages. Given that the emails were exchanged between internal and external employees, partners and students, the researcher sent an electronic consent form through email to all of the participants involved in the emails. One participant did not agree and her emails were withdrawn, 19 signed the consent form, and the rest of the participants did not reply to the email.

In order to categories the emails, the researcher noticed that some of the emails belong to chains (threads). That is, some of the emails are on-going communications between two respondents where it is hard to comprehend the content of an email in isolation. These types of messages were called chain-type messages. In fact, 359 out of the 522 collected emails (69 percent) are parts of chains. These chains usually discussed academic or organizational issues, requested information or responded to requests. In addition to these chains, it was observed that some emails did not require a reply. These email messages mainly included attachments or general interest issues and were sent to a number of recipients at the same time. These messages looked comparable to written memorandums and letters. As they did not require a reply, these messages were called solitary-type messages. The rhetorical, typographical and paralinguistic features used in the emails were examined in relation to the type of the email whether it is a chain-type or solitary-type.

\section{Rhetorical Structures}

Rhetorical structures, according to Van Dijk (1981, p.5), "may occur at all grammatical levels of the discourse. They may be defined in terms of extra structures assigned to the basic grammatical structures". Email writers mainly used four rhetorical structures that are hedges, ellipsis, substitution and repetition (see table 1).

Table 1. Linguistic and Rhetorical structures in the emails

\begin{tabular}{llll}
\hline \multicolumn{1}{c}{ Types } & $\begin{array}{l}\text { Chain-type } \\
\text { messages }\end{array}$ & $\begin{array}{l}\text { Solitary-type } \\
\text { messages }\end{array}$ & Total \\
\hline Hedges & 96 & 13 & 109 \\
Ellipsis & 75 & 17 & 92 \\
Substitution & 22 & 4 & 26 \\
Repetition & 19 & 21 & 40 \\
\hline
\end{tabular}

Examining the use of these structures in the email messages reveals that the use of hedges, ellipsis, and substitutions is very common in the chain-type messages (threads), whereas the use of repetition is the common in the solitary-type messages.

\subsection{Repetition, Ellipsis, and Substitution}

Ellipsis and substitutions are mainly used in oral communication (Halliday, 1994), whereas repetition is mainly used in written communication. According to Bloor and Bloor (1995, p. 96), speakers use ellipsis and substitution "to avoid the repetition of a lexical item and is able to draw on one of the grammatical resources of the language to replace the item". Repetition, however, "is one technique that technical writers use to establish a reliable, straightforward relationship with readers" (Zimmerman, 1983, p. 10). As such, repetition is a technique that is mainly used in professional writing and the reluctance of using this technique leads into the usage of ellipsis and substitution. The overall usage of these three features in the corpus shows that email writers tended to use oral communication techniques in their emails more than written communication techniques; however, the per-type frequency reflects a huge variation in this usage.

The usage of repetition is predominantly frequent in solitary-type messages. The writers mainly repeated the key words throughout the email in order to establish a straightforward relation with their readers and emphasize their main point. As example 1 shows, the writer of the email invites the recipients to participate in the coming conference, and informs them about the arrangements and fees. As 'the conference' is the main topic and the key word of the email, the writer repeated it eleven times throughout the email. This intensive usage of repetition in this email, in fact, meant to keep the readers focused on its main issue. The writer intended to emphasize the main point of his correspondence and present it in a clear manner. 
Example 1: All

Subject: University of Xxx Annual Administrative and Quality Conference 2010

Importance: High

Dear Colleagues

Annual Administrative and Quality Conference 2010

Following the success of the 2009 Administrative \& Quality Conference in Xxxx I am pleased to announce that the University of Xxx will be running an Administrative and Quality Conference on the $12^{\text {th }}$ and $13^{\text {th }}$ August 2010. The conference will be held over two full days at Xxxxx and will include a conference dinner on the evening of the $12^{\text {th }}$ August.

We have planned this Conference so that it incorporates a number of different areas that should prove valuable to members of staff at our Collaborative Centres who deal with administrative and quality matters. Throughout the two days we will be running various working sessions that will combine presentation and discussion groups with the help of experienced session leaders and Validation Unit staff. The Conference is an important quality and communication mechanism for the University of $\mathrm{Xxxx}$ and we would therefore expect at least one member of staff from each Institution to attend.

The Conference fee is $£ 50$ per delegate which includes the Conference sessions, lunch and refreshments over the course of the two days, as well as the Conference dinner, which will be held at the Xxxx Millennium Stadium. Institutions will be invoiced for the fee upon registration. Please note that the cost does not include overnight accommodation, however we will be providing a list of local hotels for a range of budgets.

Further details, including the timetable and session titles, will be uploaded to our University of Xxxx Conference page within due course.

We look forward seeing you at this exciting event

Best wishes

Name \& auto signature

In contrast to the usage of repetition in solitary type email messages, the writers of the chain-type email messages, as example 2 shows, intensively used ellipsis as a cohesive device. The occurrence of ellipsis in these types of messages, in fact, was mainly used to show reluctance in the writer's side to elaborate on an already known or previously mentioned issue. The writers mainly depended on the context of the email or the presupposed knowledge of the readers to interpret or possibly guess the omitted part. Noticeably, the occurrence of ellipsis in the emails took two different methods that are the dots technique (...) where the writer hints to the omitted words or clauses using a number of dots or naturally by omitting the words without a hint, as in conversations. Examining the usage of ellipsis in the emails shows that the habit of using the dots technique reflects an intentional ellipsis (omission) as a result of reluctance or unwillingness to detail the known part. The usage of natural ellipsis, however, was mainly unintentional which occurred as a result of the writing-like-speaking style. This is supported by the fact that 100 percent of ellipsis occurred in the chain-type internally exchanged emails (see example 2 below).

Example 2: 2.16. (superior, close colleague, monthly)

On Wed,June 09, 2010. at 10:36 AM,ZA wrote

Subject: Fwd: Ncc Subjects

Hi NG, How are you?

Ya, i'm really very busy with preparation for new semester...(1)so many things to do... (2)Ms. MA (Mr. VK assistant) will call you by this week to collect your timetable, material,... (3) Don't worry ...(4)Actually Mr.VK got the new materials few days ago only...(5)

sorry for the delay....(6)

Regards

ZA

The email in example 2 was sent by the head of students' counseling unit in response to a part-time lecturer requesting the material for the new semester. The sender describes her status as 'really very busy'. The writer used six ellipsis markers assuming that the recipient of the email is capable of working out the omitted words and clauses. The first two ellipses were related to the preparations for the new intake. Given that the recipient of the email is a lecturer in the Institute, the writer assumes that she knows what the staff need to do in preparation. The third ellipsis shows reluctance 
in the writer's part to continue the series of 'things' that the lecturer will be able to collect when she is called by ' $M S$. $M A$ '. As such, this ellipsis marker can be interpreted as 'etc'. In the fourth occurrence of ellipsis, the writer asked the recipient of the email to 'don't worry...'. From the context of the email, it is obvious that the writer omitted that 'you will receive your material at the right time before the semester'. In the fifth occurrence, the writer explained to the recipient the purpose of the delay telling her that ' $M r$. VK got the new materials few days ago only...' omitting what supposed to be 'this is why you didn't receive the material yet'. The last occurrence of ellipsis in this email, 'sorry for the delay... ' can be interpreted as that the writer assumes that the reader, by now, can guess why she did not reply to her email immediately and took all this time. As such, the usage of ellipsis occurred for two main purposes that are the reluctance to explain guessable issues and the reluctance to elaborate on issues that might not be of the interest of the recipient of the email. As mentioned earlier, the usage of ellipsis was constrained to the internally exchanged emails.

The usage of substitution, which is replacing "one element with another which is not a personal pronoun" (Witte \& Faigley, 1981, p. 190), as the usage of ellipsis, mainly occurred in the chain-type messages. As it is clear in example 3 , 4, 5, 6 and 7, the writers of the emails used 'one', 'ones' and 'the same' to substitute a noun, 'does' to replace a verb and 'so' to replace a clause.

Example3: I will do the correct one later

Example 4: (except the ones sent by Mr. DS)

Example 5: we should make use of the same.

Example 6: If she does, will she be able to forward to me around fifty.

Example 7: If $\underline{\text { so, }}$, see you there then.

The majority of the substitutions in the emails were used to replace a noun. In example 3 and 4, the writers used 'one' and 'ones' to replace two already mentioned nouns in the email. In example 3, the writer used 'one' to replace 'the marksheet' mentioned in the previous email. The writer submitted the marksheet previously, however, the recipient found a number of mistakes which need to be corrected. In response to the email requesting correcting the mistakes, the writer of example 3 did not use the word 'marksheet' however, replace it with 'one'. In example 7, however, the writer replaced the whole if-clause using ' $s o$ '. Returning to the email shows that 'so' refers to the possible answer of question presented earlier in the email. The sender asked the recipient 'are you [the recipient] going to attend the workshop or not? 'In order to avoid writing 'if you are coming', and to extend the semantic realm of the sentence, the writer used 'so' to replace the whole if-clause.

This shows that repetition, substitution and ellipsis were used in the two different types of messages in a way that reflects writers' evaluation of the formality and informality of the correspondence, not the medium. This can be found in the intensive usage of repetition in solitary-type email messages, which are usually sent to a group of recipients informing them about major changes or reminding them of due dates, and the usage of ellipsis in the internally exchanged chain-type messages only. This can also be found in the usage of substitution, which is as ellipsis mainly used in oral communication, in the internally and externally exchanged chain-type messages. Given that the same informants were involved in writing the different types of emails, it can be supposed that the writers conducted selfevaluation regarding the suitability of the discourse practices used for the communicative purpose and the recipient of the messages. This can be observed as the overwhelming majority of the instances using ellipsis and substitutions occurred in the emails that were exchanged internally in the Institute.

\subsection{Hedges}

Hedges are "linguistic forms which express the speaker's certainty or uncertainty about the topic under discussion" (Michael et al., 2010, p. 25). They are mainly used in oral communication (Carter, 1998); however, they are also used in written communication as well (Salager-Meyer, 1994). The actual usage of these linguistic forms functions as mitigation devices that facilitate interactions between communicators by making them more precise and compose. Examining the usage of hedges in the two different types of messages shows that they occurred in 109 emails. It is noticed that chain messages have the highest frequency of using hedges ( 88 percent of the overall occurrence).

The writers of the emails mainly used adverbial, phrasal, and generalized adjuncts hedges, in addition to idioms that show a switch in the topic. The adverbial hedges were the most common type of hedges in the emails, as the writers used adverbs such as really, very, and probably to express their certainty and doubt regarding the attributed subject. Noticeably, the adverb 'very' is the most common adverb hedge, in particular, and hedge, in general, used in the email messages. It was mainly used in front of adjectives, as in example 8, to emphasize, stress, or express a high degree of concern regarding the issue. In addition to 'very', the writers used other adverbs such as 'really' to express concern or certainty and 'probably' to express hesitation or doubt regarding the discussed matter, as it is clear in example 9.

Example 8: (3.27) We are all very worried that we are unable to contact you

Example 9: (3.120)... has to be in Singapore on 16th and I have to go to

Cairo on 17 th so I'll probably stay in KL on 16 th.

Example 10: (6.44) I guess I spoke too soon,

Example 11: (6.60) I am sure Ms BP would like know... 
In addition to the usage of adverbs, the writers also used a number of phrases such as 'I guess', as in example 10, and ' $I$ am sure', as in example 11. The use of 'I guess', does not literally reflect a 'guess'. It simply intends to weaken the effect of the upcoming sentence. The context of the email shows that the Ms. BP wrote this email to the students who asked for extensions to submit their assignments. Ms. BP, in fact, wrote to the students earlier regarding the rules and regulations of submitting assignments and that there are no extensions allowed except in the case of having 'unforeseen circumstances' and this should be supported by a signed document. However, to make her sentence less imposing and direct, she used the hedge 'I guess'. In example 11, however, the writer of the email, who is Ms. BP's assistant, used the hedge 'I am sure' for the opposite purpose of using 'I guess'. This email, in fact, was written in response to a request from a student to have an extension to submit his assignment. However, as Ms. Iffat does not have the authority to give extensions as this is Ms. BP's responsibility, she clarified this to the student emphasizing that he must have a strong reason, as Ms. BP will definitely ask about the purpose of the extension.

The third type of hedges used in the emails was what Michael et al. (2010) called generalized adjuncts hedges, which occur when using "or something, or whatever, sort of, and kind of" (Michael et al., 2010, p. 22) in a sentence. This type of hedges, in fact, was the least frequent as it occurred a single time in discussion messages (see example 12).

Example 12: so if they have not paid, or whatever, you may go after them

This email was sent by the head of studies to the head of students' counseling unit reporting the status of a number of students. The usage of 'or whatever' in the email can be interpreted as 'if they [the students] have not paid, or have not done or fulfilled any other unmentioned issue, in general, you may go after them'. The generalization in using 'or whatever' in this email gives an open option to the recipient, but reflects certainty about the action. Obviously, the head of studies expresses her desire that the head of students' counseling unit 'go after the students' if they did not pay the fees or for any other possible reason.

The fourth and final type of hedges used in the emails is the hedging using 'by the way'. The use of 'by the way' expresses a switch in the topic or an indication that the following statement or question is not related to what has been said or written earlier. The usage of 'by the way' occurred eleven times in the chain-type email messages. As example 13 shows, the writer presented a question after 'by the way'.

Example 13: (4.53) By the way, can you please let me know what's the forecasted student number?

Examining the context of the email shows that the email was sent by an external partner reminding the administrative staff of the due date to register the new students for the coming examination period. The writer took the opportunity to ask about the forecasted number of students who are going to set for the coming exam. Given that the communicative purpose of the email does not relate to the asked question, 'can you please let me know what's the forecasted student number?', the writer used the hedge 'by the way' as an indication of shifting topics and expressing the irrelevance of the coming question to the previous correspondence.

This shows that the usage of hedges in the emails functioned in accordance with Gricean maxims of quality, quantity, relevance, and manner. The writers of the emails used hedges to present what they believe to be true as in using 'we believe', We believe that Mr. Chan has informed everyone in the class...; make their statements as informative as required not more than required using 'probably'; be relevant to the topic, and even when they flouted this maxim, they openly indicated it using 'by the way'; and be clear and orderly using 'I am sure'. Therefore, it is proper to indicate that the writers of the emails did not only communicate information, but also their precise understanding of how accurate, informative, relevant, and comprehensive they are. Interestingly, the usage of hedges was particularly frequent in chaintype messages, which included a close involvement between the interactants as in discussing issues in chains or threads, requesting information or details and responding to request. The solitary-type email messages, however, had a very low rate of recurrence of hedges, which shows that the communicated information was mainly objective that did not need hedges to present its truthfulness.

\section{Paralinguistic Strategies}

Paralinguistics is "the term used to describe the tone, timbre, and intonation which accompany speech" (Sully and Dallas, 2005, P.204). Paralinguistic features, as the quotation explains, usually occur in oral communication, however, they were also present in a number of email messages. The emails, in fact, included a number of paralinguistic features such as the usage of emoticons and laughs. In effect, the corpus included twenty occurrences of emoticons and three laughs that all occurred in the chain-type email messages. The emoticons were either typed manually as in ( :) ) or chosen from the provided list of emoticons on the format of the emails as in (-)), whereas the three laughs were typed as in (hah) (see example 14).

Example 14: 2.14. Hi ZA,

Hows things? Pretty sure that you have your hands full :)

Any news on when I shall come over for material collection? ;) With less

than 3 weeks for class commencements, I am getting worried that I might

have

difficulties if the materials are going to be delivered late :D

Just give me a quick call on either my hp or house (xxxx xxxxx) yea !

Thank you.

$-\mathrm{NZG}$ 
As example 14 shows, the writer of the email, who is a part-time lecturer, used three manually typed emoticons. The three emoticons represent three different moods and their usage was either in support of the previous sentence in the email by showing lively facial expressions or in a friendly sarcastic way building on the close relationship between the interactants. However, even though the sender and the recipient of the email are close colleagues, the sender did not fancy being viewed as an assertive person especially that the recipient of the email is the direct superior of the sender. As such, the writer chose to use emoticons as a method of making the correspondence more acceptable by the recipient. As the email shows, the first emoticon, which is a smiling face ( :) ), was used after an opening sentence which shows that the writer understands that the recipient is busy. The usage of this emoticon could be interpreted as showing understanding and establishing rapport, just as the informal question 'Hows things?' at the beginning of the email. Obviously, the writer wanted to minimize the imposition. The second emoticon in the email is a wink (;)) and was used after the direct interrogative request 'any news on when...". The wink here could be interpreted as a friendly reminder with teasing that the writer of the email should have received the material for the new intake by that time. This, in fact, is also supported by the following sentence, in which the writer reminds the recipient that there are only three weeks left before the new intake commences, and that the sender needs to prepare for the coming classes. However, wishing that the recipient does not take the previous sentence as a grumble or an assertion that the superior does not carry out her job in the right manner, she used the third emoticon in the email which is a happy face (:D), indicating that no harm is meant.

This shows that emoticons were used as a supportive technique to add lively facial expressions stating the mood of the sender when writing the email and to down play any misunderstanding that might arise because of the asynchronous nature of the email. The usage of emoticons was merely practiced among close colleagues in the internally exchanged emails, which supports Bertacco and Deponte (2005) assumption that the formality and informality of the emails mainly depends on the relationship between the communicators. As the usage of emoticons, the three laughs occurred in the emails that were also internally exchanged between close colleagues to add a lively effect on the correspondence. Interestingly, the usage of emoticons and laughs was particularly frequent in chain messages; however, the overall frequency of these paralinguistic features does not exceed the 10 percent as the emoticons appeared in 20 emails and the laughs in 3 emails only. This shows that the tendency to use oral communication features was the highest in chain messages, but does not occur in a rate that enables us calling it a phenomena. Solitary-type email messages, however, were free of any paralinguistic features, which also support the tendency of viewing them as a formal type of email communication.

\section{Typographical Features}

The usage of decapitalization, different types of fonts and colors, and the occurrence of spelling mistakes are the main typographical features identified in the email messages. Decapitalization, which is the main typographical feature occurred in the emails, was distinguished to two main parts that are the decapitalization of names, days, months, and the usage of small letter after full stops, and the decapitalization of the first person pronoun ' $i$ '. Jointly, decapitalization appeared in eighty email messages. The second main typographical feature appeared in the emails is the unexpected relatively high occurrence of spelling mistakes. As figure 1 shows, the occurrence of decapitalization and spelling mistakes are the highest in chain type messages and the lowest in the solitary-type messages.

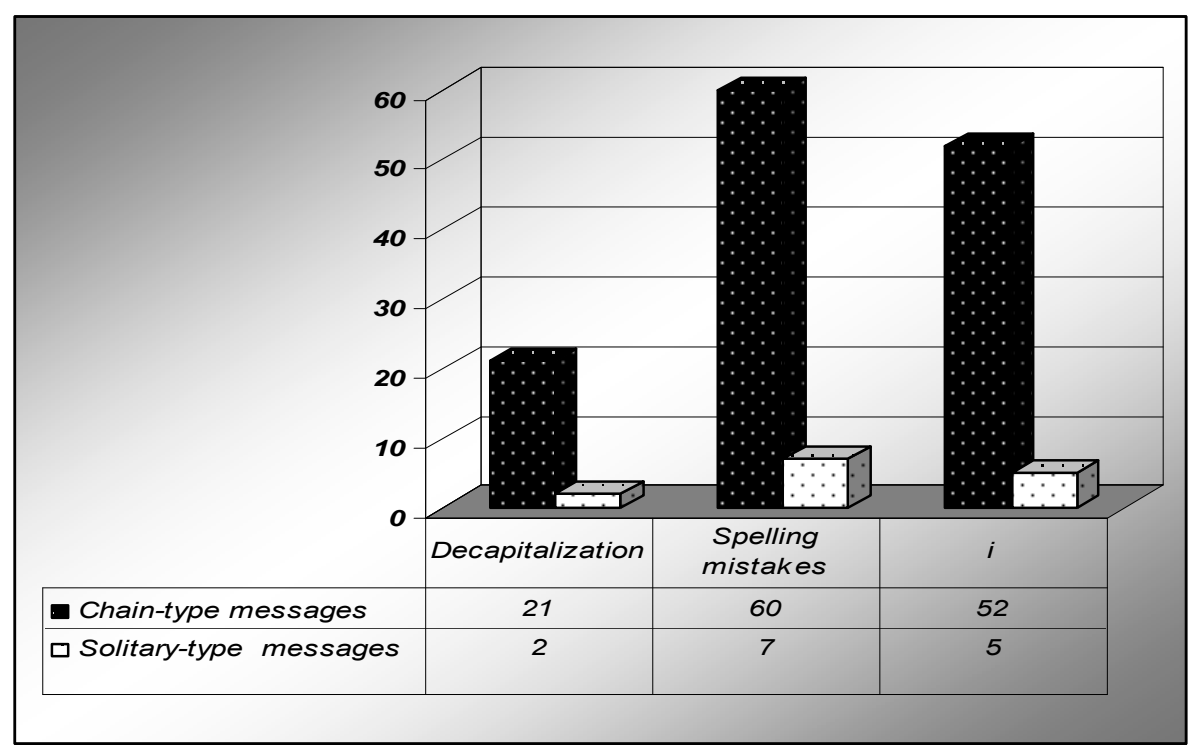

Figure 1. Typographical features used in the emails

Given that all major free email providers such as Yahoo, Hotmail and Gmail and the email provided by the Institute include an electronic 'spell check' option, which detects these types of typographical mistakes, the occurrence of these two features, in fact, reflects the reluctance in the part of the writers to electronically 'spell check' their correspondence before sending it. However, as the electronic 'spell check' option does not detect the decapitalization of the first person pronoun ' $i$ ', this also means that the writers do not even manually draft, edit or check the correspondence before 
sending it. Interestingly, the same informants, whose emails included a number of spelling mistakes and decapitalizations in their chain-type email messages, used a proper language that is free of such typographical errors in their solitary-type email messages. This shows that as a result of close involvement and the frequent exchange of emails between the same communicators the writers become reluctant to edit their emails before sending them. However, if the email was a solitary-type that does not need a reply; the writers usually edited the email before sending it.

In addition to the typographical errors, it is also found that the writers used different typographical techniques to emphasize their main points. They dark printed, italicized, underlined, capitalized all the letters and changed the color of words, sentences, and paragraphs that they want to highlight. Interestingly, these techniques were mainly used in solitary-type email messages as 20 out of the 31 dark printed and all of the six occurrences of coloring occurred when informing or updating a group of recipients about the latest updates (see example 15).

\author{
Example 15: 3.66 BUSINESS RESEARCH METHODS - VIVA - ALL ARE \\ INVITED \\ Dear Students \\ This is an Invitation for the Business Research Methods Presentation (Viva/Oral) \\ conducted on 8th May 2010 from 10am to $2 \mathrm{pm}$. \\ Group and Individual Presentations of Senior MBA Candidates who are currently \\ preparing for their dissertation proposals. \\ For Participants... (Omitted paragraphs) \\ Warm Wishes \\ Auto Signature
}

As example 15 shows, the writer capitalized the subject of the email and dark printed the date and the time of the 'VIVA' to emphasize these two points and show their special importance. According to Turnage (2007), capitalization in workplace emails is interpreted as 'shouting', however, it is noticed that capitalization in the corpus is merely used to show emphasis. This can be noticed as the type of words that were dark printed, italicized, underlined or capitalized are mainly key words build into the argument of the email. It is also found that these typographical special effects where used in the main move of the email that included the special days, months, and dates. Apparently, the British informants used these special emphasis techniques as a second reference move after the salutation of the email. The majority of these features were used by the British university in collaboration with the institute.

The usage of these features in chain-type email messages also supports the initial interpretation that they are used to emphasize or highlight a word or a sentence. As example 16, which is an extract taken from a chain-type email message, shows, the writer bolded the words 'incorrect'. The main purpose of bolding this word is that in previous email the writer wrote the same sentence in example 16, but he mistakenly typed 'correct'. In order to fix the mistake and clarify his point that 'the amount paid was incorrect' not 'correct' as stated in the previous email, he dark printed the word to emphasize and highlight the corrected version of the word.

\title{
Example 16: It should read as "I have re-reviewed these registrations
}

and found that the amount paid was indeed incorrect."

As example 15 and 16 show, the usage of these techniques occurred in the identifying topic box and/ or in the content moves of the emails. The emphasis used in the identifying topic box meant to draw the attention of the recipients to the special importance of the email, whereas the emphasis in the content moves meant to highlight the importance of the word, sentence and /or the paragraph. It is observed that the emphasis in the identifying topic move appeared in solitary messages only, whilst content emphasis occurred in chain-type messages.

\section{Discussion and Conclusion}

This study examined the rhetorical, typographical and paralinguistic features used in emails that were exchanged in a private higher educational Institute in Malaysia. Previous research on workplace email mainly examined the syntactical features of emails and compared it to spoken and written discourse (Hale and Scanlon, 1999; Yates and Orlikowski, 1993), but it did not actually look at the drive of this hybrid behavior in email communication. This study, on the one hand, examined the linguistics and paralinguistics features used in the emails, and on the other, it analyzed the purpose of using these features. This study confirmed that email communication in the workplace included spoken and written features (Cassel and Tversky, 2005; Gains, 1999). However, the occurrence of these features depended on three main factors that are the degree of involvement between the communicators, the relationship between them and the direction of the email whether it is internally or externally exchanged email.

The majority of the emails that included spoken language techniques were chain-type messages, whereas the emails that included more written language features belonged to the solitary-type email messages. That is, the use of the spoken features depended on two factors that are the degree of involvement between the communicators, on the one hand, and the frequency of the exchange, on the other. Chain-type messages were mainly sent to discuss academic or organizational issues or to request and respond to requests. In these situations, the writer composed the email and waited a response. The length of the chain depended on solving the issue. The length of the chains ranged from two to nine email messages. These types of emails included several spoken language features such as ellipsis, substitution and the 
use of hedges to show the degree of certainty and uncertainty. In the solitary-type email messages, however, the emails were mainly sent to inform the recipient about an academic or organizational issue or deliver attachments. These types of emails did not usually require a response other than thanking the sender or acknowledging receipt in times. As these email messages did not include a lot of involvement between the communicators and the frequency of exchanging emails is low, the writers mainly used written language techniques such as repetition and highlighting important information. Interestingly, the same writers, who wrote the chain-type email messages and used several spoken language features, wrote solitary-type email messages and used more written type features. This actually means that email writers were aware of the suitability or unsuitability of using certain techniques or features in a given email message.

In addition to these spoken and written features used in the emails, the emails were also rich with a number of typographical and paralinguistic features that reflect the production process of writing the emails. In addition to the use of spoken features, chain-type email messages also included lively emoticons that were either typed manually or chosen from the emoticons list provided in the formatting of the emails. Some other chain-type messages also included the use of lively laughs (hehehhee). In addition to these paralinguistic features, chain-type messages also included some typographical errors such spelling mistakes and decapitalization. Given that all major free email providers, such as Hotmail, Yahoo and Gmail and the email provided by the institute, include the spell-check option, this means that the writers sent these emails without automatically edition it, and as the automatic spell-check does not detect decapitalization of some words such as the use of (i) for (I), this also means that they did not even manually proof-read them. Interestingly, the occurrence of these features in solitary-type email messages was minimal. As such, this means that the writers of chain-type email messages paid little attention to editing or proofreading their message on contrast to the writers of the solitary-type messages. However, as the same writers, who wrote the chain-type messages and used several spoken techniques and their emails included several typographical and paralinguistic features, wrote the solitary-type messages that were written appropriately and included very little occurrences of these features, this reflects how email writers view chain-type messages as a less formal correspondence than solitary-type messages.

Having said that, it can be concluded that the hybridity of the emails depend on two main factors that are the degree of involvement and the frequency of sending emails regarding a single issue. Communication in the workplace was traditionally oral or written, however, with the introduction of the computer-mediated communication (CMC) and email, another method of communication has emerged in the workplace that is electronic communication. As electronic communication is faster and easier and does not fully fit into either of the traditional methods of communication, the users of email communication use it for the same purposes of using the traditional methods of communication. As this study revealed, the type of communication that included a lot of involvement between the communicators and high frequency of exchanging emails regarding a single issue included several spoken features. This should not be surprising as this type of communication (discussing, requesting and responding to requests) was traditionally carried out using oral methods of communication such as face-to-face or telephone conversations. The type of communication that does not include involvement and do not require frequent exchange, however, included more written features and were written properly. This also should not be surprising as the nature of these tasks (informing about issues or delivering documents) were traditionally carried out using written methods of communication in the workplace such as formal letters or written memorandums. From here, it can be said that when the communicators came across a workplace task that they want to accomplish using electronic communication, they recall their previous experience of carrying out similar tasks in the workplace traditionally. The formality and informality of the email depends on the formality and informality of the method that was used to carry out the task traditionally. The tasks that were communicated using oral methods of communication are carried out in less formal emails that include several spoken features, whereas the tasks that were traditionally carried out using written methods of communication are communicated using formal emails that include several written language features and are written appropriately.

\section{References}

AlAfnan, M. A. (2015a). Asynchronous communication: Investigating the influences of relational elements and background on the framing structure of emails. Advances in Language and Literary Studies, 6(2), 44-50. doi: 10.7575/aiac.alls.v.6n.2p.44.

AlAfnan, M. A. (2015b). Language Use in Computer-Mediated Communication: An Investigation into the Genre of Workplace Emails. International Journal of Education and Literacy Studies, 3(1), 1-11. doi: 10.7575/aiac.ijels.v.3n.1p.1.

AlAfnan, M. A. (2014a). Politeness in Business Writing: The effects of ethnicity and relating factors on mail Communication. Journal of Modern Linguistics, 4(2), 275-289. doi: 10.4236/ojml.2014.42022.

AlAfnan, M. A. (2014b, March 27-28). Interethnic workplace E-mail communication: An investigation into politeness strategies. Proceedings of the 2014 International Conference on Public Management and Education Research in Tianjin, China (iceeim-14). doi:10.2991/iceeim-14.2014.61.

Baron, N. S. (1998). Letters by phone or speech by other means: The linguistics of email. Language \& Communication, 18, 133-170. doi: 10.1016/S0271-5309(98)00005-6.

Baruch, Y. (2005). Bullying on the net: Adverse behavior on e-mail and its impact. Information \& Management, 42, 361-371. doi: 10.1016/j.im.2004.02.001 
Bertacco, M., \& Deponte, A. (2005). Email as a speed-facilitating device. A contribution to the reduced-cues perspective on communication. Journal of Computer-Mediated Communication, 10(3). Retrieved from http://jcmc.indiana.edu/vol10/issue3/bertacco.html.

Bloor, T., and Bloor, M. (1995). The functional analysis of English. London, New York: Edward Arnold.

Carter, R. (1998). Orders of reality: CANCODE, communication and culture. ELT Journal, 52(1), $43-56$. doi:10.1093/elt/52.1.43.

Case, C. J. (1996). The role of electronic messaging in the intermediate business context (interpersonal communication) (Unpublished doctoral dissertation). University of North Texas, Denton, TX.

Cassell, J., \& Tversky, D. (2004). The language of online intercultural community formation. Journal of ComputerMediated Communication, 10(2). Retrieved from http://jcmc.indiana.edu/vol10/issue2/cassell.html

Fairclough, N. (1992). Discourse and social change. Cambridge, England: Polity Press.

Fairclough, N. (1995). Critical discourse analysis: The critical study of language. London, England: Longman.

Gains, J. (1999). Electronic mail-A new style of communication or just a new medium? An investigation into the text features of e-mail. English for Specific Purposes, 18, 81-101. doi: 10.1016/S0889-4906(97)00051-3.

Gimenez, J. C. (2000). Business e-mail communication: Some emerging tendencies in register. English

for Specific Purposes, 19, 237-251. doi: 10.1016/S0889-4906(98)00030-1.

Hale, C., \& Scanlon, J. (Eds.). (1999). Wired style: Principles of English usage in the digital age. New York, NY: Broadway Books.

Halliday, M.A.K. (1994). An introduction to functional grammar (2nd ed.). London, England: Edward Arnold.

Michael, A.S., Chone, L.S., Muthusamy, C., Veeravagu, J. (2010). Gendered-linked differences in speech styles: analysing linguistic and gender in the Malaysian context. Cross-cultural communication, 6 (1), 18-28. Retrieved from http://cscanada.net/index.php/ccc/article/viewFile/1224/1298.

Palli, P., Vaara, E., \& Sorsa, V. (2009). Strategies as text and discursive practices: A genre-based approach to strategizing in city administration. Discourse and Communication, 3(3), 303-318. doi: 10.1177/1750481309337206.

Salager-Meyer, F. (1994). Hedges and textual communicative function in medical English written discourse. English for Specific Purposes, 13(2), 149-171. doi:10.1016/0889-4906(94)90013-2.

Sully, P. and Dallas, J. (2005). Essential communication skills for nursing. Edinburgh, England: Elsevier Mosby. Turnage, A. K. (2007). Email flaming behaviors and organizational conflict. Journal of Computer-Mediated Communication, 13(1), article 3. http://jcmc.indiana.edu/vol13/issue1/turnage.html.

Van Dijk, T. A. (1981). Discourse studies and education. Applied Linguistics, 2(1), 1-26. doi:10.1093/applin/II.1.1

Witte, S. P., \& Faigley, L. (1981). Coherence, cohesion and writing quality. College Composition and Communication, 32(2), 189-204. doi:10.1177/003368820003100104.

Yates, J., \& Orlikowski, W. J. (1993). Knee-jerk anti-LOOPism and other email phenomena: Oral, written, and electronic patterns in computer-mediated communication (MIT Sloan School Working Paper No. 3578-93). Cambridge, MA: MIT Sloan School of Management.

Zimmerman, I. (1983). The use of repetition in technical communication. IEEE Transactions on Professional Communication. PC-26 (1), 9-10. Retrieved from http://kmh-lanl.hansonhub.com/pc-26-9-zimmerman.pdf. 